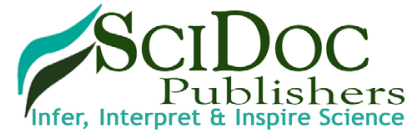

International Journal of HIV/AIDS and Research (IJHR)

ISSN 2379-1586

\title{
Management of Cardiovascular and Metabolic Alterations in HIV positive patients
}

Altizani $\mathrm{GM}^{1}$, de Oliveira $\mathrm{LD}^{1}$, dos Santos Tortajada J1 , Monteleone $\mathrm{VF}^{1}$, Mangili $\mathrm{O}^{2}$, Bonafe $\mathrm{S}^{3^{*}}$

${ }^{1}$ Medical Student, Health Sciences Department, Unicesumar, Maringá-PR, Brazil.

${ }^{2}$ Cardiology Department, Unicesumar, Maringá-PR, Brazil.

${ }^{3}$ Infectious Disease Department, Unicesumar, Maringá-PR, Brazil.

\section{Abstract}

The number of people that live with HIV continue to increase due to ample access to antiretroviral therapy (ART) that enables a higher life expectancy for the patients. The lipid changes in HIV infected patients include decrease in the high density lipoprotein (HDL) and a reduction of the serum concentration of LDL, which is associated with an increase in levels of triglycerides and very low density lipoprotein (VLDL). ART promoted great advancements in the reduction of the morbidity and mortality of individuals with HIV/AIDS. This fact contributed to a significant increase in life expectancy and quality of life of these people compared to before the development of ART. ART inherent benefits are undeniable and have been demonstrated already in various studies. However, besides the benefits, the adverse effects of these drugs are increasingly notable in each antiretroviral drug class. Metabolic disorders with lipid and glucose profile alterations were found in most antiretroviral drug classes, especially in the protease inhibitors (PIs). Such alterations result in an increased prevalence of cardiovascular diseases (CVD), therefore should be analyzed with criteria in all individuals that receive antiretroviral treatment. Cardiovascular risk stratification scores are heavily used and despite being useful they present limited capability, especially because they do not consider the HIV infection. The treatment of the lipid profile modifications must be primarily based in life style changes, diet and physical exercise and when persistent, pharmacotherapy, notably the statins, should be utilized.

Keywords: HIV; Dyslipidemia; Antiretroviral Therapy; Cardiovascular Diseases; Statins.

\section{Introduction}

According to the World Health Organization (WHO), HIV/AIDS prevalence by the end of 2014 was of 36.9 million people. Out of this amount, about 1.2 million (4\%) died due to the disease, being that in developing countries, $20 \%$ of the deaths were attributed to cardiovascular events caused by the virus, by the antiretroviral therapy as an adverse effect and by know factors that increase cardiovascular risk like obesity, sedentary life style, smoking and genetic predisposition [1].

Highly active antiretroviral therapy (HAART) access expansion and the reduction in the incidence of HIV infection resulted in an accentuated decrease in worldwide mortality related to the virus. In 2013 there was a 35\% decrease in deaths caused by HIV when compared to the year of 2005 and an expressive increase in life expectancy. In a study done in North America and Europe, published in 2008, the patient that started HAART at 20 years old had an increase in life expectancy from 56 years old in 1999 to 69 years old in 2005 [2].

Although there is a significant increase in life expectancy of the patients, there is still a high rate of new infections and of death related to the virus. To reduce the morbidity and mortality of this disease and stop the HIV epidemic, UNAIDS presented a set of goals known as 90-90-90, in which it was established that until the year of $2020,90 \%$ of people infected by HIV should know their serologic status; $90 \%$ of people with a HIV/ AIDS diagnosis should receive antiretroviral therapy; and $90 \%$ of people undergoing treatment should have a undetectable viral load, therefore incapable of transmitting the virus [3].

The number of people that live with HIV continue to increase due to ample access to antiretroviral therapy (ART) which increases



Citation: Altizani GM, de Oliveira LD, dos Santos Tortajada J, Monteleone VF, Bonafe S (2016) Management of Cardiovascular and Metabolic Alterations in HIV positive patients. Int J AIDS Res. 3(7), 110-118. doi: http://dx.doi.org/10.19070/2379-1586-1600022

Copyright: Bonafe $\mathbf{S}^{\circ}$ 2016. This is an open-access article distributed under the terms of the Creative Commons Attribution License, which permits unrestricted use, distribution and reproduction in any medium, provided the original author and source are credited. 
the life expectancy of these patients [4]. According to UNAIDS in June 2001, only one million people had access to ART. In June 2015, this number has increased to more than 15.8 million, also increasing the patient undergoing treatment's life quality (LQ). LQ in conjunction with treatment of AIDS patients and pharmacological innovations allows for expansion of a patient's life span and their wellbeing [5].

Not withstanding the benefits of HARRT far outweigh the potential risks, it is a fact that treatment can result in disorders related to adhesion to treatment and toxicity [6]. Studies show that specific types of HAART toxicity added to the effects of HIV infection alone are capable to inducing the development of lipoatrophy/dystrophy and metabolic syndrome (dyslipidemia, insulin resistance associated or not with type 2 diabetes (DM2) and increase in blood pressure), being this factor associated with an increase cardiovascular disease risk (coronary artery disease and cerebrovascular accident) $[6,7]$.

Therefore, these metabolic alterations are described as multifactorial, including the effects of HIV infection and the toxicity of specific types of antiretroviral therapy, specially the protease inhibitors (PIs), changes in the body and also including factors that increase the atherosclerotic risk in patients not infected by HIV like genetic factors, diet, use of alcohol, smoking tobacco, obesity and sedentary life style $[6,7]$.

Regarding the metabolic disorders that occur in HIV infected patients, it was found that the main ones are dyslipidemia, that are alterations in the lipids' and lipoproteins' levels, and lipodystrophy. Lipodystrophy are a heterogenic group of adipose tissue disorders characterized by selective alteration of fat in various body parts. The association of fat redistribution alteration can happen in conjunction with metabolic alterations, being this pattern similar to the one found in the "metabolic syndrome". According to clinical protocol and treatment guidelines for the management of HIV infection in adults, lipodystrophy has an important impact in the LQ of people living with HIV/AIDS, causing them physical, psychological and social problems [6].

Two important studies were done to verify the interferences of HAART in patients infected by HIV. The START (Strategic Timing of Antiretroviral Treatment) study, initiated in March 2011, was conducted by the International Network for Strategic initiatives and Global HIV trials (INSIGHT) in 215 places in 35 countries. The study involved 4,685 men and women. Infected by HIV 18 years or older, being the average age 36 years old. The participants had never received ART and were recruited with a CD4 cell count within the normal range - above 500 cells by cubic millimeter (cells $/ \mathrm{mm}^{3}$ ). Approximately half of the participants of the study were randomized to initiate antiretroviral therapy immediately (early treatment) and the rest were randomized to delay the beginning of therapy until their CD4 cell count was of 350 cells $/ \mathrm{mm}^{3}$ or less. In average, the participants of this study were accompanied during three years [8]. This study showed the benefits of early start of the HAART, reporting reductions in more than $40 \%$ in the risk of death or in developing AIDS defining diseases, compared to the start of treatment when the CD4 limits were reached $\left(<350\right.$ cells $/\left(<350\right.$ cells $\left./ \mathrm{mm}^{3}\right)$. However, a notable secondary finding of this study was that the immediate start of HAART did not stop the development of cardiovascular diseases (CVD), what seems to contradict various evidences that, especially those of the SMART (Strategies for Management of Antiretroviral Therapy) study [9].

In the SMART study, approximately 5000 patients infected with HIV undergoing an established ART, with a CD4 cells count of 350 or more for continued HAART were randomized against individuals that interrupted treatment until the CD4 cell count was below 250, when HAART was restarted. Without a doubt, the most informative result of the SMART study was not that treatment interruptions increase the risk of opportunistic infections or mortality but, that patients that interrupt HAART had an increase of approximately $70 \%$ in the risk of cardiovascular complications [9].

Moreover, the mains cardiovascular events occurred 5 times more frequently than opportunistic infections in people that interrupted treatment. Another important consideration established in this study was that specific antiretroviral drug classes, like the protease inhibitors, can be associated with a higher CDV relative risk when compared to other classes.

Besides the cases related to cardiovascular diseases like Cerebrovascular Accident (CVA) and Acute Myocardial Infarction (AMI), there is scientific evidence, presented in the Conference on Retroviruses and Opportunistic Infections (CROI) 2016, that the increase of triglycerides and cholesterol secondary to HIV is associated with worsening of the neurocognitive condition in virus carriers older than 50 years of age. This data associated to all metabolic disorders mentioned previously, corroborate the importance of constantly doing lipid and glucose control of the patient, to promote better quality of life [10].

\section{HIV vs. Dyslipidemias/Lipodistrophys}

The lipid changes in patient infected by HIV include decrease of high density lipoprotein (HDL) levelsand reduction of the serum concentration of LDL, associate to an increase in the triglycerides and very low density lipoprotein (VLDL) levels [7]. This finding characterizes the phenotypic profile of the metabolic syndrome, extremely atherogenic due to, among other reasons, the presence of lipoprotein residuals of VLDL and LDL, small and dense [11]. The effect is amplified by the loss of the inflammation modulation capacity that HDL normally performs in a healthy organism [7].

There are also relevant correlations between the increase in plasmatic viral RNA, TCD4+ lymphocyte cell count, the decrease of HDL and LDL and the increase in VLDL. The smaller the TCD4 lymphocyte count, the bigger is the triglycerides concentration and smaller are the levels of total cholesterol and LDL [12, 13]. However, low HDL concentrations are found independent of the TCD4+ lymphocyte cell count [13][14].

Lipoproteins rich in triglycerides, like chylomicrons, that are synthetized in the intestines, and HLD cholesterol, formed in the liver, are secreted into the blood plasma. HIV-1 contributes to the decrease in HDL when it alters a mediator of export of excess cholesterol from macrophages, the ATP Binding Cassette transporter A1 (ABCA-1) $[15,16]$.

Moreover, inflammation caused by the virus stimulates endothelial lipase and phospholipase A2 that diminishes plasma levels of 
HDL [17]. Additionally, HDL becomes rich in triglycerides in situations like hypertriglyceridemia, becoming a substrate more avid for hepatic lipase, resulting in the lipoprotein dysfunction.

HIV associated dyslipidemia is very similar to the one observed in other chronic infections. Elevated levels of Gama-Interferon in advanced HIV are related to the increase in plasma triglycerides due to the decrease in clearance of lipoproteins rich in triglycerides. Similarly, the Tumor necrosis factor $\alpha(\mathrm{TNF} \alpha)$ and interleucin-6 (IL6) are elevated in patients not using HAART and increase even more during opportunistic infections. TNF $\alpha$ interferes with the metabolism of free fatty acids and with lipid oxidation, reduces insulin mediated lipolysis suppression, causes and increase in the platelets activation and also in the production of reactive oxygen species [18].

The patient with HIV also suffers from protein loss and weight loss that can contribute to the plasma HDL and LDL reduction [15].

The HIV virus itself is responsible for causing alterations in the lipid profile in about $50 \%$ of cases, even before the beginning of HAART. This way, the collateral effect of HAART caused dyslipidemia is more pronounced, therefore increasing cardiovascular risk of people living with HIV/AIDS (PLHA) [19].

Furthermore, there are studies that indicate that $7 \%$ of patients infected with HIV, that have not started HAART, presented with cardiovascular alterations. Among the ones described, it was noted that infection by HIV, opportunistic diseases, cancer and nutritional deficiencies (vitamin B12 deficiency) cause mainly alterations in the myocardium, endocardium and pericardium [20].

Besides dyslipidemia, HAART can also cause other alterations, like insulin resistance, hyperglycemia and adipose tissue redistribution. This group of alterations is known as HIV lipodystrophy Syndrome (HIVLS). It is important to note that the virus itself is capable of inducing these alterations. However, this phenomenon has been observed more frequently with HAART use, especially with the older drugs [21-24].

The virus' direct action in $\beta$ pancreatic cells alters the regulation function of the insulin secretion mechanisms. It contributes to the glucose intolerance and metabolic syndrome [25].

HIV lipodystrophy it is characterized by the redistribution of body fat in HIV positive patients. The clinical manifestations of lipodystrophy are divided in 3 classes: (1) lipoatrophy, (2) lipohypertrophy and (3) mixed [26]. The first body alteration perceived were the accumulation of fat tissue in the abdominal region and in the dorsocervical region, also known as buffalo hump [27]. With regards to the lipoatrophy, there is loss of peripheral subcutaneous tissue. The upper and lower limbs become thinner, the skin becomes attenuated, which allows for visualization of the superficial vasculature, giving the patient a pseudo athletic physique [27].

In lipohypertrophy there is anaccumulation of fat tissue in a central or localized region, like abdomen, breasts, dorsocervical regions and others regions in a localized manner. The abdomen of a patient with lipohypertrophy presents with a distended abdomen and the adipose tissue is deposited in the intra-abdominal region, viscerally, or in between them. It is observed also an increase in intra-abdominal pressure, which predisposes the patient to abdominal hernias [27].

Lipohypertrophy occurs mainly in patients with advanced age during the beginning of HAART, with a high BMI and those using protease inhibitors [28]. It was observed that lipodystrophy's prevalence in patients using PI is of $64 \%$ in men and $10.5 \%$ in women $[23,29]$. The breasts' increased volume in the female patient with lipodystrophy is due to the fat component without mandatory association with glandular hypertrophy. Gynecomastia, in which there is glandular hypertrophy, and pseudogynecomastia, when there is only adipose tissue accumulation, can both occur in male patients [27].

Metabolic alterations encompass lipid alterations and changes in the glucose cycle. Such alterations can be associated or not to anatomic deformities $[30,31]$. In male patients the accumulation of adipose tissue was observed in the upper thorax and axillary region and also occurred in both genders in the anterior cervical region and suprapubic region [32]. However HIV associated lipodystrophy is marked, mainly, by inferior lipodystrophy, especially in the lower limbs. In these cases there are also alterations in the upper body, however to a lesser degree [7].

With regards to bone metabolism it is still not clear if the loss of mineral bone density is a component of the lipodystrophy syndrome [32]. One of the complications of HIVLS is avascular necrosis, by hyperlipidemia and the HIV virus pathogenicity considered risk factors for femoral head osteonecrosis [33].

A 12 month long prospective analyzes of 366 patients infected by HIV showed that, from the beginning of HAART therapy, there was a cumulative incidence of $29 \%$ for lipoatrophy, $23 \%$ for lipohypertrophy and $13 \%$ for mixed lipodistrophy. The study reinforces the hypothesis that anatomic alterations and lipid alterations are common among individuals that initiated antiretroviral therapy for the first time. In this analyzes, the use of PIs was associated with all types of lipodystrophy [34].

Independent of etiology, the factors that promote physiopathological alterations in body fat in patients infected by HIV promote cellular metabolism alterations and simultaneously inhibit normal fat storage in favor of ectopic deposition of visceral fat $[35,36]$. The final phenotype, being it lipoatrophic or lipohypertrophic, probably depends on the base line quantity of body fat as well as on the total energy balance. Those with more total body fat before HAART and a positive energy balance can present new increase in axial body fat including in the breasts, dorsocervical region and visceral, while those with less total body fat and negative energy balance can present with a decrease of body fat in limbs and face, without ectopic fat deposition (meaning visceral fat) $[35,37]$. More importantly, however, is that the final phenotype, independent of the initial stages (total fat gain or total fat loss), consistently results in a proportional increase in central adiposity [35].

\section{HIV/AIDS and HAAR'T}

According to the World Health Organization (WHO) the antiretroviral therapy shave the potential to reduce morbidity and 
mortality was well as improve LQ of people living with HIV/ AIDS (PLHA) [38, 39].

HAART is essential for the treatment of people infected by HIV. Each class of drugs acts in different stages of the retrovirus life cycle therefore stopping viral replication, maintaining the TCD4+ lymphocytes count stable and a the viral load undetectable [39].

However, these drugs, not different than most, are also responsible for producing side effects. In adults the treatment with HAART is associated with metabolic alterations like lipodystrophy, insulin resistance, hyperglycemia and dyslipidemia, with consequent elevation of atherosclerosis risk [40].

In the elderly, other health complications, besides the ones described above, can be the resultof the interaction of HAART with medications utilized to treat other conditions. This is particularly true for the elderly that have many comorbidities and tend to take two or more medications for different conditions concomitantly. Besides the fact that long exposition to HAART increases risks for cardiac disease, heart attacks and obesity, new research suggests that certain combinations of HAART can cause abnormal bone metabolism, pancreatitis, peripheral neuropathy and lactic acid accumulation. Various types of cancer, like Hodgkin's lymphoma, leukemia, melanoma, mouth cancer, lung cancer among others, have a significantly higher incidence in elderly HIV positive individuals when compared to the general population [41].

Children and teenagers infected by HIV that are treated with HAART can have significant improvement in clinical results, nutritional status and life quality [42]. However HAART has also been associated with undesirable side effects in children, like gastrointestinal intolerance, mitochondrial toxicity, lipodystrophy, dyslipidemia, retinol and beta-carotene deficiency, therefore being high risk for CVD [43, 44].

In pregnant women infected by HIV it is recommended treatment with antiretroviral to prevent perinatal transmission of HIV and maintain the mother's health. Although the benefits of HAART during pregnancy are uncontestable, adverse effects, including pre-eclampsia, premature labor and low birth weight are increasingly associated with HAART, especially the protease inhibitors, which hypothetically contribute to alterations in the levels of progesterone, causing the adverse effects [45].

Currently, six classes of antiretroviral drugs are available in the market: nucleoside reverse transcriptase inhibitors (NRTI); Nonnucleoside reverse transcriptase inhibitor (NNRTI); Protease inhibitors (PI); Fusion inhibitors (FI) Integrase inhibitors (II) and Entry inhibitors (EI) [46]. Among those, PI, NRTI and NNRTI are the most frequently associated, in HIV positive patients, with lipodystrophy and alterations in the lipid and the glucose metabolism [42].

Protease Inhibitors (Pi): Mechanism Of Action, Glucose Alterations, Lipid Alterations and Side

\section{PIs and mechanism of action}

PIs (Atazanavir, Darunavir, Fosamprenavir, Indinavir, Lopinavir,
Ritonavir, Saquinavir e Tipranavir) are considered the third antiretroviral drugs class [46-48]. In patients infected with HIV, various studies identified that the IPs are the drugs responsible for the most side effects in the lipid and glucose profile when compared to other classes of ART [47].

The general mechanism of action of PIs results in the blockage of the protease enzyme, stopping its action, interrupting the production of new copies of HIV infected cells [46].

\section{PIs and glucose alterations}

The prevalence of hyperglycemia and diabetes mellitus is significantly higher in the population that uses HAART than in the general population. Insulin resistance (IR) is common in HIV positive individuals, mainly among those that receive PIs and among those that developed lipoatrophy or visceral fat accumulation. Data from the Multicenter AIDS Cohort Study (MACS) showed a risk of developing DM four times higherin HIV positive individual than in those not infected by HIV [6]

Therapies containing PIs, alter the glucose metabolism by two distinct mechanisms. The first is due to the drug direct effect and the second occurs as a result of long term therapy, which leads to lipodystrophy. PIs act directly in the induction of peripheral insulin resistance in the skeletal muscle and adipose tissue, causing deterioration in the beta cells ability to compensate these alterations. It can also act directly in the GLUT-4, hindering glucose homeostasis [6].

The IPs as a drug class, however, doesnot interfere equally in the glucose elimination. In studies done with rat adipocytes all glucose capture was inhibited, being the drug used APV (amprenavir), RTV (ritonavir) or the more adequate therapeutic combination LPV (lopinavir) with RTV. However there was no inhibition with the use of ATV (atazanavir) when compared with controls in therapeutic concentrations in a euglycemic state [35].

This data are of utmost importance seeing that hyperglycemia is a risk factor for various pathologies, including CVD [6].

\section{PIs and lipid alterations}

Carr and cols developed a theory that relates homology among the proteins involved in the lipid metabolism: CRABP-1 (Cytoplasmic Retinoic-Acid Binding Protein Type 1) and LRP (Low Density Lipoprotein-Receptor Related Protein) and the place where the protease inhibitor binds, its catalytic site. Based on this theory, IPs inhibit important stages of human metabolism since they inhibit the action of CRABP-1 and bind to LRP, what diminishes Apo B-100 rich lipoprotein capture, resulting in hyperlipidemia [49, $50]$.

When IPs occupied CRBP-1's binding site, it interrupts retinoic acid metabolization and decrease activity of PPAR- $\gamma$ (Peroxisomeproliferator-activated receptor type gamma). This receptor is important in the differentiation and apoptosis of adipocytes and improves the peripheral insulin sensitivity. These occurrences lead to hyperlipidemia by the reduction of peripheral storage and increase in the lipid liberation into the blood stream $[49,50]$.

Studies show that not only the inflammatory state due to HIV 
infection, but also the treatment with IPs are responsible by the trigger that deflagrates the answers to cellular stress, leading to adverse effects in the intermediate metabolism. The physiological result is the creation of a decrease in the adipocytes total storage capacity and the remaining ones become resistant to additional fat storage. Therefore, the lipid metabolites in excess in the circulation that were normally absorbed by the adipose tissue are deposited ectopically in lean tissue (muscles and liver), where it hinges insulin action. This process takes a pathological cycle of lipotoxicity and lipoatrophy and a clinical phenotype of fat distribution with a high waist-hips ratio, similar to the metabolic syndrome [35].

\section{PIs and the side effects}

Indinavir: Even though it is not a medication that is amply used nowadays, in studies done with individuals not infected with HIV, indinavir, when evaluated for a period of four weeks, promoted an increase in the glucose levels and a reduction of $20 \%$ in insulin sensibility. Schwarz and cols also presented a reduction of $17 \%$ in insulin sensibility with an increase in the production of glucose and in glycogenolysis, showing that indinavir induces peripheral insulin resistance and increase the production of endogenous glucose. It is noted that this state might be associated with the HIV infection, probably due to the virus direct action that interferes with the function of the beta pancreatic cells or in the insulin's mechanism of action [49].

Lopinavir/ritonavir (Lop/R): With the objective of determining the effects of Lop/ $\mathrm{R}$ in the physical performance and body composition, WONG et al., (2013) did studies in overweight older rats. Initially they evaluated the animals' prehensile strength before and after seven weeks of treatment with Lop/R. These rats, compared to the control group, showed a decrease in their prehensile strength in the same time period, resulting in a significant difference of $14 \%$ between the two groups. Other parameters analyzed the maximum velocity reached by the rats in a rotarod. The ones that received Lop/R obtained substantially inferior velocities than the control animals. Therefore, with the obtained data it was concluded that long term treatment with Lop/R can be prejudicial to physical strength and can reduce the level of physical and metabolic activity of its users [51].

Lop/ $\mathrm{R}$ and other PIs can cause an acute but reversible inhibition of glucose capture stimulated by insulin. To evaluate the effects of the long term use of the drug on insulin resistance, insulin tolerance and glucose tests were done in animals. Both the control and animals treated with Lop/R had similar fasting glucose concentrations (150-165 mg/dL) and non-fasting (175-189 mg/ dL). Therefore, it was identified that Lop/R does not affect glucose and insulin tolerance. These results are also compatible to clinical observations in humans [51].

There was an increase of liver weight in rats treated with Lop/R but triglycerides concentrations both in liver and serum were similar in both groups. These results is somewhat unexpected based on previous studies in rodents, but compatible with clinical reports that showed that more than $90 \%$ of patients that take Lop/R did not have an important increase of lipids level $(<12 \%)$ [51].

Darunavir/ritonavir: Ritonavir boosted darunavir was generally well tolerated in sick patients with HIV-infections in clinical trials, being most adverse events non-severe. In a analyzes of 48 weeks, the side effects associated with one or two daily doses of darunavir/ritonavir were diarrhea, nausea, headache, nasopharynx infections. The most common effect in patients submitted to treatment included increase of triglycerides and total cholesterol $[52,53]$.

Considering the protease inhibitor that were analyzed, it was concluded that most, some more than others, are capable of inducing undesirable alterations in the lipid profile of HIV infected patients. In particular, dyslipidemia occurs approximately in $70 \%-80 \%$ of patients with HIV receiving HAART and it has been associated with all the available PIs. Given the current necessity of life long therapy, long term toxicity considerations, such as hyperlipidemia and lipodystrophy, are becoming more important when considering different treatments. New drugs, so to have a higher probability of finding a place in HIV treatment, must have a high bioavailability, low resistance, more potency, few side effects and a low rate of long term complications [54, 55].

Atazanavir (ATZ): Atazanavir is a drug release in 2004 and is characterized by being a potent and safe azapeptide PI with a high inhibitory quotientand a pharmacokinetic profile that allows for a daily single dose administration. In contrast with other PIs, ATZ is not associated with an increase in total cholesterol, LDL and triglycerides level. Therefore, in patients with lipid profile alterations using other PIs, the change to AZT is recommended as a viable option to improve the atherogenic lipid profile, maintaining virological control [56].

In this regard, the multiple mechanismsof the PIs in the development of lipid and glucose abnormalities should always be considered when choosing treatment in an individual bases because each patient's characteristics like age, gender, tobacco use, family history of cardiovascular diseases and high total cholesterol, can be determinants for the severity of metabolic alterations of patients and, consequently, for their quality of life $[42,47]$.

\section{Nucleoside Reverse Transcriptase Inhibitors (NRTI)}

The NRTIs lead to cellular mitochondrial toxicity by inhibiting DNA-polymerase $\gamma$, an enzyme responsible for mitochondrial DNA synthesis and that acts regulating the respiratory chain. The thymidine analogues (zidovudine - AZT and stavudine - d4T), are involved with the reduction of peripheral fat, what can cause lipoatrophy, since the mitochondrial toxicity alter adipogenesis and lipogenesis, increasing also the oxidative stress and the production of adiponectin, leptin and proinflammatory cytokines (IL-6, monocyte chemotactic protein 1 - MCP1) [48, 57].

The NRTIs reduce lipid reserves, leading to the development of insulin resistance (IR) in addition to the main clinical findings, the presence of peripheral lipoatrophy and triglycerides level alterations. Others NRTIs, although can also lead to mitochondrial toxicity mediated cellular damage, like didanosine (peripheral neuropathy, pancreatitis and hyperlactatemia) and tenofovir (renal tubular toxicity), are not associated with adipose tissue toxicity [48]. 
The effects of treatment with tenofovir indicate the lipid lowering action of this NRTI, which makes it different from other drugs belonging to the same substitution class. Its use can be a useful strategy to improve dyslipidemic patient's lipid profile, especially triglycerides levels and consequently there is a reduction of cardiovascular risk for HIV infected children. The change from stavudine to tenofovir is virologically and immunologically safe and allows for a significant improvement in the lipid profile [48].

\section{Non - Nucleoside Reverse Transcriptase Inhibitor (NNRTI)}

Traditionally, the NNRTIs are not considered to be lipodystrophy causing agents. Recent data associates efavirenz (EFZ) with lipoatrophy due to the decrease in the SREBP-1 (sterol regulatory element - binding protein 1) expression [57].

These data explain Souza's (2013) affirmation, which indicates that lipid profile alterations are not as evident in patients utilizing a combination of tenofovir and lamivudine in comparison to those using zidovudine and lamivudine, stavudine and lamivudine or didanosine and lamivudine with the finding of lower LDL-C, total cholesterol and triglycerides serum concentration.

Recent information from D:A:D: (Data Collection on Adverse Events of Anti-HIV Drugs Study) reinforce the theory that HAART therapy with NNRTI is associated to a modest increase in triglycerides levels, however do not increase LDL-C and do not cause a reduction in the HDL-C levels $[6,58]$.

\section{Cardiovascular Risk Stratification}

The D:A:D: multi-cohort study investigates the incidence of cerebrovascular and cardiovascular accidents in HIV infected patients with the association of cardiovascular disease risk factors and HAART. A paper published in 2007 by the group confirmed the existence of an association between the duration of antiretroviral therapy and an increase risk of suffering myocardial infarction with an adjusted relative risk of 1.16 per year of exposition to therapy ( $\{$ IC 95\% $\%: 1.09-1,23)$. The incidence of MI was of $1.53 / 1.000$ people-year for those who were not exposed to PIs and of 6.01/1.000 people year for those exposed to PIs. This study indicated an increase of $26 \%$ in the estimated myocardial infarction per year of HAART containing PI exposition [49].

Clinical scores are very utilizes to determine the cardiovascular risk being the Framingham score one of the most used to identify cardiovascular risk and it uses blood pressure, total cholesterol or LDL-cholesterol to do the calculations. Age, tobacco use, diabetes and HDL-cholesterol are alsovariables considered in the calculations. There is a table specific for men and one for women [59].

However, although they are very useful, clinical scores when used alone present a limited cardiovascular risk stratification capacity in a significant portion of the general population. In fact, these limitations are even more significant in younger patients and women. It is in this context that some laboratorial and image tests, among them the coronary calcium score (CS), can perform an important role as a complementary tool to the clinical scores in the risk stratifications of asymptomatic patients [60].
In a study with HIV positive patients there was no significant association between and elevated Framingham score and a positive calcium score. Therefore, it can be inferred that Framingham score is not the best tool to determine the cardiovascular risk in this specific population [61].

Atherosclerosis detected by the calcium score was significantly higher only in patients that were undergoing antiretroviral therapy for at least 8 years, which is the time period for this disease's development [61].

Another cardiovascular risk marker that has still to be carefully evaluated in HIV infected patients is the apoB/apoA1 ratio. The Ding et al., 2014 study with a non HIV infected population showed that an increase in this ratio is associated with an increased risk for cardiovascular diseases. It has been suggested that the apoB/ apoA1 ratio can be considered a tool to adjust the risk evaluation and therefore the therapy objectives. This ratio can be valuable to determine the risk or the response to treatment of HIV infected patients $[62,63]$.

It is easily notable that the current cardiovascular risk scores do not consider HIV infection and antiretroviral therapy use, being that the HIV positive individuals are a population that possesses a disproportionate atherosclerotic risk and lipid levels due to the inflammatory process imminent to the viral infection.

\section{Nonpharmacologic Therapy}

The nonpharmacologic treatment measures must be the first option, meaning, changes in life style, including following a diet plan, an exercise plan and if the metabolic alterations persist, initiate pharmacologic therapy, however with extreme caution. [49].

Omega-3 supplementation initially showed some improvement of the lipid profile, however recent meta-analysis showed that there is no benefit with regards to a decrease in cardiovascular events, coronary events and mortality [64].

Life style modifications, including diet and exercise, presented a significant reduction of lipodystrophy [65]. In general it can be verified that exercise has a benefic effect in improving lipid parameters and in central adiposity [66].

Randomized controlled studies need to be developed to elucidate the nutritional treatment of dyslipidemias, lipodistrophy, insulin resistance and high systemic blood pressure in PLHA using HAART [65].

Silva et al., 2014 observed that the group that consumed food considered "good" presented high HDL levels in comparison to the group that consumed "bad" food. The results showed the importance of diet and adequate ingestion as a protection factor and concluded that an unbalanced diet increases the risk for cardiovascular diseases in HIV positive patients [67].

There is a lack of studies that evaluate the effect of physical training and adequate nutrition with the estimated coronary risk in patients infected with HIV that use HAART. A study analyzed 
a prevention program composed by controlled diet, physical training and cessation of tobacco use with the coronary risk estimation in 10 years. It was observed that after 6 months of the programs establishment there was a reduction form $92 \%$ to $27.6 \%$ of the coronary risk in patient with high cardiovascular risk [68].

\section{Pharmacological Therapy}

The benefic effects of statins in the cholesterol metabolism in the general populationare already confirmed. Statins significantly reduce total cholesterol (TC), LDL and triglycerides that ate strong independent predictors of cardiovascular events. This reduction can improve the cardiovascular prognosis of these patients. Combined with nonpharmacologic interventions like diet and commutations in antiretroviral therapy, even though there is a pharmacokinetic interaction risk (especially with PIs), statins and antiretroviral therapy are efficient and safe when simultaneously administered, representing an important part of the dyslipidemia treatment [69].

The most critical aspect of statin use in HIV positive patients is the safety of its administration combined with antiretroviral therapy, due to innumerous interaction between these drugs. PIs inhibit the CYP3A4 enzyme what increases the serum concentration of the statin, especially simvastatin and lovastatin, elevating the drug toxicity risk. Nonetheless, NNRTIs are CYP3A4 inductors and can diminish plasmatic concentrations of the statins [69].

In case of concomitant administration of statins and antiretroviral therapy, the monitoring of renal function, liver enzymes and creatine phosphokinase (CPK) is fundamental due to the strengthening of the nephrotoxic, hepatotoxic and myotoxic effects of these medications [49]. Some studies with HAART did no show a significant interaction with statins in relation to adverse effects, corroborated by the fact that use of statins with adequate dose control and efficient monitoring can be used in a safe manner [69].

In patients that use ART, statins prescribe for primary prevention present a good security profile and were efficient in improving dyslipidemia. Rosuvastatin showed vast reductions of TC, LDL and triglycerides and a significant increase of HDL. Pravastatin slightly diminished the levels of TC, LDL and triglycerides but it was not capable of significantly increasing HDL. Atorvastatin showed controversial safety results and while there is no new evidence, its prescription and use must be rigorously monitored. Sinvastatin, even though it is safe and efficient, should not be concomitantly prescribed with PIs, due to pharmacological interactions. Fluvastatin seems to be safe and efficient, although the results were based in a single study [69].

Due to difficulties of attaining the lipid goals in patients with $\mathrm{HIV}$, the use of combined therapy must be considered. In clinical practice, the fibrates are added to the treatment when the triglyceride levels are too high or, more frequently, when the monotherapy with statin did not reach good results. The combination of fibrates and statins is included in the guidelines for the general population as well as for HIV positive patients, however, in this last group, there must be awareness about the increased risk for side effects [70]. Domingos et al., affirmed that the pharmacological profiles of ciprofibrate and rosuvastatin are adequately efficient in reducing triglycerides and total cholesterol, are adequately safe and tolerable, being considered effective in controlling dyslipidemia secondary to HAART [71].

Combined therapy with fenofibrate and pravastatin for HIV related dyslipidemia, provides substantial improvements in the lipid parameters and it appears to be safe. The International AIDS society USA panel recommends pravastatin and atorvastatin as first choice agents [72].

Ezetimibe is an efficient and safe drug for the reduction of lipids in dyslipidemia in HIV positive patients, being able to be used in patients with a weak response to statins. Moreover, the combination of statin and ezetimibe is efficient and safe in these patients [72]. According to Saeedi et al., the association of ezetimibe and rosuvastatin was well tolerated, with improvement in the safety profile and biochemistry, with low drug interaction probability between ezetimibe and ART. This treatment also obtained reduction of the apoB/ apoA1 ratio. Grandi et al., affirms that the 6 month treatment with ezetimibe and fenofibrate is well tolerated and as efficient as pravastatin in the reduction of LDL cholesterol. It also shows the capability of reducing triglycerides level and increasing HDL, commonly unaltered with a pravastatin monotherapy [70].

\section{Conclusion}

Presently, it is verified that HIV infection and the use of ART can contribute to an increase in the cardiovascular risk of patients infected by HIV. Various studies are done about the theme however new research is necessary to elucidate the metabolic alterations that occur by the HIV intrinsic inflammatory process and by the side effects of ART. Additionally, cardiovascular risk stratification scores currently do not contemplate HIV infection as an additional risk factor for cardiovascular diseases. This should be implemented, once the infection leads to an atherosclerotic risk disproportional to the lipid levels due to the installed inflammatory process. A more detailed and accurate knowledge about the cardiovascular risk of these patients will allow for a better treatment plan, being it life style changes with controlled diet and exercise or the use of statins in monotherapy or in association with other drugs, aiming for a better quality of life for these patients and less morbidity and mortality.

\section{References}

[1]. Zaaqoq AM, Khasawneh FA, Smalligan RD (2015) Cardiovascular Complications of HIV-Associated Immune Dysfunction. Cardiology Research and Practice 2015:8.

[2]. (2008) Life expectancy of individuals on combination antiretroviral therapy in high-income countries: a collaborative analysis of 14 cohort studies. The Lancet 372(9635): 293-9.

[3]. UNAIDS. (2014) 90-90-90 - AN AMBITIOUS TREATMENT TARGET TO HELP END THE AIDS EPIDEMIC: Joint United Nations Programme on HIV/AIDS (UNAIDS).

[4]. (2015) AIDS by the numbers 2015. UNAIDS

[5]. Ferreira BE, Oliveira IM, Paniago AMM (2012) Qualidade de vida de portadores de HIV/AIDS e sua relação com linfócitos CD4+, carga viral e tempo de diagnóstico. Revista Brasileira de Epidemiologia 15:75-84.

[6]. (2013) Guia De Consulta Rápida Protocolo Clínico E Diretrizes Terapêuticas Para Manejo Da Infecção Pelo Hiv Em Adultos. Ministério da Saúde (BR) Secretaria de Vigilância em Saúde Departamento de DST, AIDS e Hepatites Virais.

[7]. Grunfeld C. (2010) Dyslipidemia and its Treatment in HIV Infection. Top 
HIV Med 18(3):112-8.

[8]. (2015) Starting Antiretroviral Treatment Early Improves Outcomes for HIV-Infected Individuals.

[9]. Siedner MJ (2016) START or SMART? Timing of Antiretroviral Therapy Initiation and Cardiovascular Risk for People With Human Immunodeficiency Virus Infection. Open Forum Infectious Diseases 3(1):ofw032.

[10]. Mukerji SS. (2016) Lipid Profiles and APOE4 Allele Impact Midlife Cognitive Decline in HIV+ Men on ART. Conference on Retroviruses and Opportunistic Infections (CROI); Massachusetts General Hospital, Boston, MA, United States.

[11]. Xavier HT, Izar MC, Faria Neto JR, Assad MH, Rocha VZ, et al., (2013) V Diretriz Brasileira de Dislipidemias e Prevenção da Aterosclerose.

[12]. Grunfeld C, Kotler DP, Hamadeh R, Tierney A, Wang J, et al., (1989) Hypertriglyceridemia in the acquired immunodeficiency syndrome. Am J Med 86(1):27-31.

[13]. Nguemaïm N, Mbuagbaw J, Nkoa T, Alemnji G, Této G, et al., (2010) Serum lipid profile in highly active antiretroviral therapy-naïve HIV-infected patients in Cameroon: a case-control study. HIV Med 1;11(6):353-9.

[14]. Adewole OO, Eze S, Betiku Y, Anteyi E, Wada I, et al., (2010) Lipid profile in HIV/AIDS patients in Nigeria. Afr Health Sci 10(2):144-9.

[15]. Oh J, Hegele RA. (2007) HIV-associated dyslipidaemia: pathogenesis and treatment. The Lancet Infectious Diseases 7(12):787-96.

[16]. Mujawar Z, Rose H, Morrow MP, Pushkarsky T, Dubrovsky L, et al., (2006) Human immunodeficiency virus impairs reverse cholesterol transport from macrophages. PLoS Biol 4(11):e365.

[17]. Rader DJ. (2006) Molecular regulation of HDL metabolism and function: implications for novel therapies. J Clin Invest 116(12):3090-100.

[18]. Ducobu J, Payen MC. (2000) Lipids and AIDS. Rev Med Brux 21(1):11-7.

[19]. Green ML. (2002) Evaluation and Management of Dyslipidemia in Patients with HIV Infection. Journal of General Internal Medicine 17(10):797-810.

[20]. Guimarães MMM, Machado LJC, Vieira AC, Andrade ACS, Souza BN, et al., (2013) Atherosclerotic cardiovascular disease and HIV infection: an update. Rev méd Minas Gerais 23(4):464-73.

[21]. Behrens G, Dejam A, Schmidt H, Balks H-J, Brabant G, et al., (1999) Impaired glucose tolerance, beta cell function and lipid metabolism in HIV patients under treatment with protease inhibitors. AIDS 13(10):63-70.

[22]. Carr A, Cooper DA (2000) Adverse effects of antiretroviral therapy. The Lancet 356(9239):1423-30.

[23]. Valente AMM, Reis AF, Machado DM, Succi RCM, Chacra AR. (2005) Alterações metabólicas da síndrome lipodistrófica do HIV. Arquivos Brasileiros de Endocrinologia \& Metabologia 49:871-81.

[24]. Montessori V, Press N, Harris M, Akagi L, Montaner JSG. (2004) Adverse effects of antiretroviral therapy for HIV infection. Canadian Medical Association Journal 170(2):229-38.

[25]. Dubé MP. (2000) Disorders of glucose metabolism in patients infected with human immunodeficiency virus. Clin Infect Dis 31(6):1467-75.

[26]. Thiébaut R, Daucourt V, Mercié P, Ekouévi DK, Malvy D, et al., (2000) Lipodystrophy, metabolic disorders, and human immunodeficiency virus infection: Aquitaine Cohort, France, 1999. Groupe d'Epidémiologie Clinique du Syndrome d'Immunodéficience Acquise en Aquitaine. Clin Infect Dis 31(6):1482-7.

[27]. (2009) Handbook of facial lipoatrophy treatment: recommendations for facial fling with polymethylmethacrylate in HIV/Aids patients. Ministério da Saúde (BR) Secretaria de Vigilância em Saúde Departamento de DST, AIDS e Hepatites Virais. Available from: http://www.aids.gov.br/sites/default/files/man_lipoatrofia03-web.pdf.

[28]. Finucane KA, Archer CB (2010) Dermatological aspects of medicine: highly active antiretroviral therapy and the treatment of human immunodeficiency virus. Clinical and Experimental Dermatology 35(1):107-9.

[29]. Gervasoni C, Ridolfo AL, Trifirò G, Santambrogio S, Norbiato G, et al., (1999) Redistribution of body fat in HIV-infected women undergoing combined antiretroviral therapy. AIDS 13(4):465-71.

[30]. Soares FMG, Costa IMC (2011) Lipoatrofia facial associada ao HIV/AIDS: do advento aosconhecimentos atuais. Anais Brasileiros de Dermatologia 86:843-64.

[31]. Collins E, Wagner C, Walmsley S (2000) Psychosocial impact of the lipodystrophy syndrome in HIV infection. AIDS Read 10(9):546-51.

[32]. Gkrania-Klotsas E, Klotsas A-E (2007) HIV and HIV treatment: effects on fats, glucose and lipids. British Medical Bulletin 84(1):49-68.

[33]. Behrens GMN, Stoll M, Schmidt RE (2000) Lipodystrophy Syndrome in HIV Infection. Drug Safety 23(1):57-76.

[34]. Madge S, Kinloch-de-Loes S, Mercey D, Johnson MA, Weller IVD. (1999) Lipodystrophy in patients naive to HIV protease inhibitors. AIDS 13(6):735.

[35]. Flint OP, Noor MA, Hruz PW, Hylemon PB, Yarasheski K, et al., (2009) The role of protease inhibitors in the pathogenesis of HIV-associated lipodystrophy: cellular mechanisms and clinical implications. Toxicol Pathol
37(1):65-77.

[36]. Unger RH (2003) Minireview: weapons of lean body mass destruction: the role of ectopic lipids in the metabolic syndrome. Endocrinology 144:515963.

[37]. He Q, Engelson ES, Kotler DP. (2005) A comparison of abdominal subcutaneous adipose tissue pattern in obese and lean HIV-infected women. J Nutr 135(1):53-7.

[38]. WHO. Number of deaths due to HIV/AIDS. Global Health Observatory (GHO) data: World Health Organization 2015.

[39]. Gill CJ, Griffith JL, Jacobson D, Skinner S, Gorbach SL, et al., (2002) Relationship of HIV Viral Loads, CD4 Counts, and HAART Use to HealthRelated Quality of Life. Journal of Acquired Immune Deficiency Syndromes 30:485-92.

[40]. Domingos H, Cunha RVd, Paniago AMM, Souza ASd, Rodrigues RL, et al., (2012) Rosuvastatina e ciprofibrato no tratamento da dislipidemia em pacientes com HIV. Arquivos Brasileiros de Cardiologia 99:997-1007.

[41]. Sean Cahill RV (2013) Growing Older With HIV/AIDS: New Public Health Challenges. Am J Public Health 103(3):e7-e15.

[42]. Souza SJ, Luzia LA, Santos SS, Rondó PHC (2013) Lipid profile of HIVinfected patients in relation to antiretroviral therapy: a review. Revista da Associação Médica Brasileira 59:186-98.

[43]. Battistini TR, Sarni RO, de Souza FI, Pitta TS, Fernandes AP, et al., (2010) Lipodystrophy, lipid profile changes, and low serum retinol and carotenoid levels in children and adolescents with acquired immunodeficiency syndrome. Nutrition (Burbank, Los Angeles County, Calif) 26(6):612-6.

[44]. Tremeschin MH, Sartorelli DS, Cervi MC, Negrini BVdM, Salomão RG, et al., (2011) Nutritional assessment and lipid profile in HIV-infected children and adolescents treated with highly active antiretroviral therapy. Revista da Sociedade Brasileira de Medicina Tropical 44:274-81.

[45]. Papp E, Mohammadi H, Loutfy MR, Yudin MH, Murphy KE, et al., (2015) HIV Protease Inhibitor Use During Pregnancy Is Associated With Decreased Progesterone Levels, Suggesting a Potential Mechanism Contributing to Fetal Growth Restriction. The Journal of Infectious Diseases 211(1):10-8

[46]. Quais são os antirretrovirais Portal sobre AIDS, doenças sexualmente transmissíveis e hepatites virais.

[47]. Hejazi N, Rajikan R, Kwok Choong CL, Sahar S (2013) Metabolic abnormalities in adult HIV infected population on antiretroviral medication in Malaysia: a cross-sectional survey. BMC Public Health 13:758.

[48]. (2013) Protocolo Clínico e Diretrizes Terapêuticas Para Manejo da Infecção pelo HIV Em Adultos. Ministério da Saúde (BR) Secretaria de Vigilância em Saúde Departamento de DST, AIDS e Hepatites Virais.

[49]. Kramer AS, Lazzarotto AR, Sprinz E, Manfroi WC (2009) Alteraçóes metabólicas, terapia antirretroviral e doença cardiovascular em idosos portadores de HIV. Arquivos Brasileiros de Cardiologia 93:561-8.

[50]. Carr A SK, Chisholm D, Cooper A (1998) Pathogenesis of HIV-1-protease inhibitor-associated peripheral lipodystrophy, hyperlipidaemia, and insulin resistance. The Lancet 352:1881-3.

[51]. Wong S, Bhasin S, Serra C, Yu Y, Deng L, et al., (2013) Lopinavir/Ritonavir Impairs Physical Strength in Association with Reduced Igf1 Expression in Skeletal Muscle of Older Mice. Journal of AIDS \& clinical research 4:216.

[52]. McKeage K, Perry CM, Keam SJ (2009) Darunavir: a review of its use in the management of HIV infection in adults. Drugs 69(4):477-503.

[53]. Pham PA, la Porte CJL, Lee LS, van Heeswijk R, Sabo JP, et al., (2009) Differential Effects of Tipranavir plus Ritonavir on Atorvastatin or Rosuvastatin Pharmacokinetics in Healthy Volunteers. Antimicrobial Agents and Chemotherapy 53(10):4385-92.

[54]. Troll JG (2011) Approach to Dyslipidemia, Lipodystrophy, and Cardiovascular Risk in Patients with HIV Infection. Curr Atheroscler Rep Feb; 13(1): 51-6.

[55]. (2007) We're taking care of you - A Helpful Guide to HIV and Metabolic Complications. Department of Health and Human Services (US) - National Institute of Allergy and Infectious Diseases.

[56]. Möbius U, Lubach-Ruitman M, Castro-Frenzel B, Stoll M, Esser S, et al., (2005) Switching to atazanavir improves metabolic disorders in antiretroviral-experienced patients with severe hyperlipidemia. J Acquir Immune Defic Syndr 39(2): 174-80.

[57]. Lipodistrofia Portal sobre AIDS, Doenças Sexualmente Transmissíveis e Hepatites Virais: Departamento de DST, Aids e Hepatites Virais.

[58]. Law MG, Friis-Møller N, El-Sadr WM, Weber R, Reiss P (2006) The use of the Framingham equation to predict myocardial infarctions in HIV-infected patients: comparison with observed events in the D:A:D Study. HIV Med 7(4):218-30.

[59]. Lotufo PA (2008) O escore de risco de Framingham para doenças cardiovasculares. Rev Med (São Paulo) 87(4):232-7.

[60]. Azevedo CF, Rochitte CE, Lima JAC (2012) Escore de cálcio e angiotomografia coronariana na estratificação do risco cardiovascular. Arquivos Bra- 
sileiros de Cardiologia 98:559-68.

[61]. Monteiro VS, Lacerda HR, Uellendahl M, Chang TM, Albuquerque VMd, et al., (2011) Escore de cálcio na avaliação da aterosclerose em pacientes com HIV/AIDS. Arquivos Brasileiros de Cardiologia 97:427-33.

[62]. Saeedi R, Johns K, Frohlich J, Bennett MT, Bondy G (2015) Lipid lowering efficacy and safety of Ezetimibe combined with rosuvastatin compared with titrating rosuvastatin monotherapy in HIV-positive patients. Lipids in Health and Disease 14(1):1-8.

[63]. Ding D, Li X, Qiu J, Li R, Zhang Y, et al., (2014) Serum Lipids, Apolipoproteins, and Mortality among Coronary Artery Disease Patients. BioMed Research International 2014:11.

[64]. Xavier HT, Izar MC, Faria Neto JR, Assad MH, Rocha VZ, et al., (2013) V Diretriz Brasileira de Dislipidemias e Prevençăo da Aterosclerose. Arq bras cardiol 101(4,supl.1):1-20.

[65]. Falco M, Castro AdCdO, Silveira EA. (2012) Terapia nutricional nas alteraçóes metabólicas em pessoas vivendo com HIV/aids. Revista de Saúde Pública 46:737-46.

[66]. Mullen A, Loonam CR. (2012) Nutrition and the HIV-associated lipodystrophy syndrome. Nutrition Research Reviews 25:267-87.

[67]. Silva TALdS, Barboza RR, Andrade RDd, Medeiros RCdSCd, Medeiros JAd, et al., (2014) Relationship between dietary intake and use of protease inhibitors with anthropometric and biochemical parameters of lipodystrophy in people living with HIV. Nutr Hosp 30(4):935-40.

[68]. Lazzarotto AR, Pereira FB, Harthmann AdA, Bazzo KO, Vicenzi FL, et al., (2014) Treinamento físico no risco de doença isquêmica cardíaca em sujeitos HIV/AIDS em uso de TARV. Revista Brasileira de Medicina do Esporte 20:233-6.

[69]. Gili S, Grosso WM, D'Ascenzo F, Lonni E, Calcagno A, et al., (2016) Comparative safety and efficacy of statins for primary prevention in human immunodeficiency virus-positive patients: a systematic review and metaanalysis. European Heart Journal.

[70]. Grandi AM, Nicolini E, Rizzi L, Caputo S, Annoni F, et al., (2014) Dyslipidemia in HIV-positive patients: a randomized, controlled, prospective study on ezetimibe+fenofibrate versus pravastatin monotherapy. Journal of the International AIDS Society 17(1):19004.

[71]. Domingos H, Cunha RVd, Paniago AMM, Souza ASd, Rodrigues RL, et al., (2012) Rosuvastatina e ciprofibrato no tratamento da dislipidemia em pacientes com HIV. Arquivos Brasileiros de Cardiologia 99:997-1007.

[72]. Husain NE, Ahmed MH (2015) Managing dyslipidemia in HIV/AIDS patients: challenges and solutions. HIV/AIDS (Auckland, NZ) 7:1-10. doi:10.2147/HIV.S46028. 